\title{
FADS Gene Polymorphisms, Fatty Acid Desaturase Activities, and HDL-C in Type 2 Diabetes
}

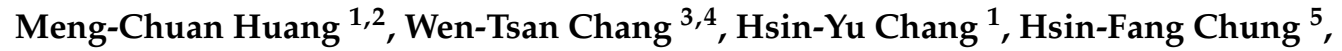 \\ Fang-Pei Chen ${ }^{6}$, Ya-Fang Huang ${ }^{7}$, Chih-Cheng Hsu ${ }^{7,8, *}$ and Shang-Jyh Hwang ${ }^{9}$ \\ 1 Department of Public Health and Environmental Medicine and Graduate Institute of Medicine, \\ College of Medicine, Kaohsiung Medical University, Kaohsiung 80708, Taiwan; \\ mechhu@kmu.edu.tw (M.-C.H.); s3223251720@gmail.com (H.-Y.C.) \\ 2 Department of Nutrition and Dietetics, Kaohsiung Medical University Hospital, Kaohsiung 80756, Taiwan \\ 3 Department of Surgery, School of Medicine, College of Medicine, Kaohsiung Medical University, \\ Kaohsiung 80708, Taiwan; wtchang@kmu.edu.tw \\ 4 Division of General and Digestive Surgery, Department of Surgery, Kaohsiung Medical University Hospital, \\ Kaohsiung 80756, Taiwan \\ 5 School of Public Health, University of Queensland, Brisbane, Queensland 4006, Australia; \\ evelyn150@yahoo.com.tw \\ 6 Department of Nutrition and Health Sciences, College of Health Sciences, Chang-Jung Christian University, \\ Tainan 71101, Taiwan; w787fanny@gmail.com \\ 7 Institute of Population Health Sciences, National Health Research Institutes, 35 Keyan Road, Zhunan, \\ Miaoli County 35053, Taiwan; avon@nhri.org.tw \\ 8 Department of Health Services Administration, China Medical University, Taichung 40402, Taiwan \\ 9 Division of Nephrology, Department of Internal Medicine, Kaohsiung Medical University and University \\ Hospital, Kaohsiung 80708, Taiwan; sjhwang@kmu.edu.tw \\ * Correspondence: cch@nhri.org.tw; Tel: +886-37-246-166
}

Academic Editor: William Chi-shing Cho

Received: 16 April 2017; Accepted: 20 May 2017; Published: 28 May 2017

\begin{abstract}
Polyunsaturated fatty acids (PUFA) correlate with risk of dyslipidemia and cardiovascular diseases. Fatty acid desaturase (FADS) single nucleotide polymorphisms (SNPs) modulate circulating PUFA concentrations. This study examined influence of FADS1 and FADS2 genetic variants on desaturase activities and blood lipid concentrations in type 2 diabetes patients, and further assessed their interrelationships. Selected SNPs (FADS1: rs174547, rs174548, rs174550; FADS2: rs174575, rs174576, rs174583, rs498793 and rs2727270) were genotyped in 820 type 2 diabetes patients and compared with those reported in the HapMap. Patient subgroups $(n=176)$ without taking lipid-lowering medicine were studied to assess influence of tag SNPs including rs174547, rs174575, rs498793 and rs2727270 on delta-5 desaturase (D5D: 20:4 (n-6)/20:3 (n-6)) and delta-6 desaturase (D6D:18:3 (n-6)/18:2 (n-6)) activities, and blood lipids. FADS1 rs174547 TT/TC/CC and FADS2 rs2727270 CC/CT/TT were significantly ( $p$ for trend $<0.05$ ) associated with reduced HDL-C, D5D and D6D activities. Upon adjustment for confounders, D5D $(p=0.006)$ correlated significantly and D6D marginally $(p=0.07)$ correlated with increased HDL-C levels, whereas rs174547 and rs2727270 polymorphisms were not associated. D6D andD5D activities may play a role in modulating HDL-C levels in type 2 diabetes. Future studies with larger sample sizes are needed to investigate how FADS genetic variations interact with desaturase activities or PUFAs in the metabolism of lipoproteins in diabetic patients.
\end{abstract}

Keywords: diabetes; FADS1; FADS2; HDL; genetic polymorphisms; polyunsaturated fatty acids 


\section{Introduction}

Type 2 diabetes mellitus is a well-established risk factor for cardiovascular diseases, and is frequently accompanied by metabolic abnormalities including hypertriglyceridemia, high low-density lipoprotein cholesterol (LDL-C) or low high-density lipoprotein cholesterol (HDL-C) [1,2]. Recent studies have implicated a role of raising HDL-C for the prevention and management of diabetes $[2,3]$. Adults with diabetes have heart disease-related mortality rates that are two to four times higher than those without diabetes [4]. It is, therefore, important to study risk factors for the development of dyslipidemia in patients with type 2 diabetes.

Fatty acid desaturase 1 (FADS1) and fatty acid desaturase 2 (FADS2) genes are located closely on chromosome 11 (11q12-13.1) [5] and encode delta-5 desaturase (D5D) and delta-6 desaturase (D6D) to [6] serve as rate limiting enzymes for catalyzing the formation of double bonds at delta- 5 and delta- 6 positions in n-6 or n-3 long chain polyunsaturated fatty acids (PUFA), respectively [7]. Recent gene and genome-wide association studies (GWAS) have shown that polymorphisms in the FADS gene cluster have an important impact on lipid profiles and glucose homeostasis, [8-10] and thus they play a role in modifying risk of metabolic diseases.

Two recent genome wide association studies have also associated several common variants of FADS1 and FADS2 genes with polygenic dyslipidmia [11,12]. Several studies suggested that a cluster of SNPs including rs174547, rs174576 and rs174537are in strong linkage disequilibrium (LD) within the FADS1-FADS2 region in Caucasian and Asian adults [13-15]. SNPs of FADS1 rs174547 or FADS2 rs174576 have been correlated with LDL-C [10], HDL-C [11,14] and triglyceride [14]. Furthermore, these polymorphisms also have been shown to modulate D5D and D6D activities, further leading to changes in n-3/n-6 PUFA status [16,17] in healthy adults of different ethnicities.

FADS genotypes and desaturase indices have been implicated in insulin resistance [18] and heart diseases [19]. To date, the influences of common FADS genetic variants on desaturase activities and blood lipids profile have only been studied in healthy adults of Caucasian or Asian origin, and little research has been undertaken in individuals with diabetes. In this study, we genotyped SNPs (FADS1: rs174547, rs174548, rs174550; FADS2: rs174575, rs174576, rs174583, rs498793 and rs2727270) in 820 patients with type 2 diabetes and compared our findings with those reported in HapMap in a healthy population. We further assessed influence of tag SNPs including rs174547, rs174575, rs498793 and rs2727270 on D5D and D6D activities and blood lipids in a subgroup ( $n=176)$ who were not recorded as receiving lipid lowering drugs on medical records.

\section{Subjects and Methods}

\subsection{Study Subjects and Inclusion Criteria}

We enrolled type 2 diabetes patients who participated in the diabetes management through an integrated delivery system (DMIDS) (ClinicalTrials.gov NCT00288678). Patients between 30 and 70 years old who had been diagnosed or newly diagnosed as having type 2 diabetes by their physicians of primary healthcare clinics based on criteria established by the American Diabetes Association (ADA) were recruited [20]. Pregnant women and participants with a history of myocardial infarction, cerebrovascular accident (e.g., stroke), foot amputation, and uremia under dialysis were excluded at baseline recruitment. The protocol of the study has been described previously [21]. Briefly, 1209 newly diagnosed type 2 diabetes patients were recruited between August 2003 and December 2005. Of those patients, 874 completed the follow-up through the end of 2009. We only included 820 participants who had available DNA collected in year of 2008 for the genotyping that we performed in this study. Furthermore, we selected 176 diabetes patients who, according to their medical records, had not used lipid-lowering drugs (statin and fibrate) and had complete plasma fatty acid workups to examine the associations of concentrations of fatty acids with different polymorphisms of FADS. The protocol of this study was approved by the Ethics Committee of the National Health Research Institutes and Kaohsiung Medical University Hospital, Taiwan. Each subject signed an informed written consent form. 


\subsection{Clinical Data Collection and Measurements}

Trained research assistants or nurses measured height, weight, as well as systolic and diastolic blood pressure. Venous blood was collected after overnight fasting for at least $8 \mathrm{~h}$ for hematological analysis. All blood samples were stored at $2-8{ }^{\circ} \mathrm{C}$ and delivered to a central laboratory certified by the College of American Pathology and US Commission on Office Laboratory Accreditation. Glycated hemoglobin ( $\mathrm{HbA1c}$ ) was assessed by high-performance liquid chromatography (Variant II; Bio-Rad Laboratories, Hercules, CA, USA). Levels of plasma blood glucose, triglycerides, cholesterol, LDL-C, HDL-C, and serum creatinine were measured by an automatic analyzer (Hitachi 7060; Hitachi High Technologies, Tokyo, Japan).

\subsection{Gas Chromatography Analysis of Plasma Lipids}

Extraction of plasma lipids was based on a method described by Bligh and Dyer [22]. Extracted lipids were derivatized to fatty acid methyl esters using boron trifluoride-methanol. These were quantified by gas chromatography (6890 N GC system, Agilent Technologies, Santa Clara, CA, USA). The gas chromatographer was equipped with a Agilent J\&W DB-225 capillary column $(30 \mathrm{~m} \times 0.25 \mathrm{~mm}$ inner diameter $\times 0.25 \mathrm{~mm}$ film) with $\mathrm{N}_{2}$ being employed as the carrier gas. Fatty acids levels were measured using C17:0 (Sigma, St. Louis, MO, USA) as internal standard and fatty acid concentrations were expressed as a percentage of total area under the peaks (weight \%) [23].

\subsection{Measurements of Desaturase and Pathway Activities}

Desaturase and pathway activities were evaluated by ratios of product/precursor fatty acids. Desaturase activities for D6D and D5D were as follows: D6D activity $=[18: 3(n-6) / 18: 2(n-6)]$ and D5D activity $=[20: 4(n-6) / 20: 3(n-6)]$ [24]. Pathway activities were as follows: $n-3$ pathway $=[20: 5(n-3) / 18: 3$ $(\mathrm{n}-3)]$ and $\mathrm{n}-6$ pathway $=[20: 4(\mathrm{n}-6) / 18: 2(\mathrm{n}-6)][15]$.

\subsection{SNP Selection and Genotyping}

FADS1 SNPs (rs174547, rs174548, rs174550) and FADS2 SNPs (rs174575, rs174576, rs174583, rs498793 and rs2727270) were chosen based on findings of previous studies [10,11,15]. Four of the SNPs including rs174547, rs174575, rs2727270 and rs498793were tag SNPs with minor allele frequencies (MAF) $>5 \%$ and were reported in the HapMap of the Han Chinese healthy population. Pairwise I $\mathrm{D}^{\prime} \mid$ and $r^{2}$ values between SNPs were computed using Haploview version 4.2 (Broad Institute, Cambridge, MA, USA). The four SNPs (rs174548, rs174550, rs174576, and rs174583) were genotyped in our study and found to be highly correlated with the tag SNP (rs174547) $\left(r^{2} \geq 0.9\right)$. Thus, only the results for the tag SNP (rs174547) are presented.

FADS SNP genotyping was analyzed by the GenomeLabTM SNPstream ${ }^{\circledR}$ genotyping platform (Beckman Coulter Inc., Fullerton, CA, USA) and its accompanying SNPstream software suite were used to perform multiplex polymerase chain reaction (12-plex PCR) and SNP genotyping by methods previously described [25]. Primers were designed to amplify DNA, and probes designed to identify the SNPs. Ten percent of the samples were duplicated, masked and tested twice. All SNPs were found to be accurately genotyped at a rate of $>95 \%$.

\subsection{Statistical Analysis}

All statistical operations were performed using SPSS version 21.0 (SPSS Inc., Chicago, IL, USA). The normality of the continuous variables was performed using Kolmogorov-Smirnov test. If data were not normally distributed, parameters were transformed logarithmically before analysis. Biochemical data among the FADS1 and FADS2 SNP genotypes were compared for significance using independent $t$-test and analysis of variance (ANOVA). Simple linear regression analysis was used to evaluate the trend among FADS SNP genotypes ( $p$ for trend). Multiple linear regression analysis was used to evaluate independent associations among FADS polymorphisms, desaturase (D5D and D6D) activities 
and blood lipids levels by adjusting for confounding factors (age, gender, body mass index, education ( $\leq 6$ year, $>6$ year), and diabetes duration (years), $\mathrm{HbA1c}$, alcohol use (yes, no) and exercise (yes, no)). We used the Haploview version 4.2 (Broad Institute, Cambridge, MA, USA) to calculate the pairwise $\left|\mathrm{D}^{\prime}\right|$ and $r^{2}$ values between SNPs. All statistical results were considered significant at $p$ value $<0.05$ in a two-sided test.

\section{Result}

\subsection{General Characteristics of Type 2 Diabetes Patients}

The distributions of demographic and clinical characteristics of all type 2 diabetes patients $(n=820)$ and those who did not use lipid-lowering drugs $(n=176)$ are summarized in Table 1 . The mean age of the subjects was 59.5 years old and average diabetes duration was 11.0 years. A little more than half $(52.6 \%, n=431)$ were female.

Table 1. Baseline characteristics of 820 type 2 diabetes patients and those who without using lipid-lowering drugs $(n=176)^{\mathrm{a}}$.

\begin{tabular}{|c|c|c|}
\hline Variables & $\begin{array}{l}\text { All Type } 2 \text { Diabetes Patients } \\
\qquad(n=820)\end{array}$ & $\begin{array}{l}\text { Type } 2 \text { Diabetes Patients without Using } \\
\text { Lipid-Lowering Drugs }(n=176)^{\mathrm{b}}\end{array}$ \\
\hline \multicolumn{3}{|l|}{ Clinical Measures } \\
\hline Age (year) & $59.5 \pm 8.5$ & $58.3 \pm 8.9$ \\
\hline Female $(\%)$ & $431(52.6)$ & $99(56.3)$ \\
\hline Diabetes duration (year) & $11.0 \pm 5.7$ & $10.7 \pm 5.3$ \\
\hline Education $\leq 6$ years $(\%)$ & $446(54.4)$ & $90(51.1)$ \\
\hline Smoking $(\%)$ & $243(29.6)$ & 47 (26.7) \\
\hline Drinking (\%) & $99(12.1)$ & $21(11.9)$ \\
\hline Exercise (\%) & $529(64.5)$ & $108(61.4)$ \\
\hline Body mass index $\left(\mathrm{kg} / \mathrm{m}^{2}\right)$ & $26.1 \pm 3.8$ & $25.4 \pm 3.9$ \\
\hline Systolic blood pressure (mmHg) & $136.4 \pm 19.6$ & $135.5 \pm 22.6$ \\
\hline Diastolic blood pressure (mmHg) & $79.4 \pm 11.0$ & $79.1 \pm 11.3$ \\
\hline Triglyceride $(\mathrm{mmol} / \mathrm{L})$ & $1.8 \pm 1.1$ & $1.6 \pm 1.1$ \\
\hline Cholesterol (mmol/L) & $4.9 \pm 0.9$ & $4.8 \pm 0.9$ \\
\hline LDL-C $(\mathrm{mmol} / \mathrm{L})$ & $3.1 \pm 0.8$ & $3.0 \pm 0.8$ \\
\hline HDL-C (mmol/L) & $1.0 \pm 0.3$ & $1.0 \pm 0.3$ \\
\hline non-HDL-C (mmol/L) & $3.9 \pm 0.8$ & $3.8 \pm 0.9$ \\
\hline Fasting glucose (mmol/L) & $8.3 \pm 2.7$ & $8.7 \pm 3.0$ \\
\hline Hemoglobin A1c (\%) & $8.0 \pm 1.5$ & $8.2 \pm 1.6$ \\
\hline Serum creatinine $(\mu \mathrm{mol} / \mathrm{L})$ & $86.6 \pm 67.7$ & $80.7 \pm 63.2$ \\
\hline Blood urea nitrogen $(\mathrm{mmol} / \mathrm{L})$ & $5.8 \pm 2.8$ & $5.3 \pm 2.3$ \\
\hline \multicolumn{3}{|l|}{ Plasma PUFA $(\%)^{\mathrm{c}}$} \\
\hline Total PUFA & & $40.5 \pm 3.5$ \\
\hline n-3 PUFA & & $6.9 \pm 2.2$ \\
\hline C18:3 n3 (alpha-linolenic acid) & & $0.9 \pm 0.3$ \\
\hline C20:5 n3 (eicosapentaenoic acid) & & $0.9 \pm 0.7$ \\
\hline n-6 PUFA & & $33.6 \pm 3.6$ \\
\hline C18:2 n6 (linoleic acid) & & $24.1 \pm 4.1$ \\
\hline $\mathrm{C} 18: 3 \mathrm{n} 6$ & & $0.2 \pm 0.2$ \\
\hline C20:3 n6 & & $1.7 \pm 0.5$ \\
\hline C20:4 n6 (arachidonic acid) & & $6.6 \pm 1.6$ \\
\hline
\end{tabular}

Abbreviations: LDL-C, low density lipoprotein cholesterol; HDL-C, high density lipoprotein cholesterol. PUFA, polyunsaturated fatty acids; ${ }^{a}$ Data presented was mean \pm SD or $\mathrm{n}(\%) .{ }^{\mathrm{b}} 176$ type 2 diabetes patients without using lowering lipid drugs (statin and fibrate) and with complete plasma fatty acid measures were selected. ${ }^{c}$ Only selected $n-6$ and n-3 PUFA were presented.

\subsection{Genotype and Allele Distribution of FADS1 and FADS2 Polymorphisms among All Type 2} Diabetes Patients

Table 2 shows the distributions of genotype and allele frequencies of the eight selected SNPs in diabetes patients. None of the genotype distributions in our study were differed significantly from those expected under Hardy-Weinberg equilibrium (HWE), except for rs498793. For all SNPs examined 
in our diabetic subjects, the frequencies of genotypes, rs174547, rs174548, rs174576, rs174583 and rs498793, were comparable to those reported in the HapMap CHD (Chinese in Metropolitan Denver, $\mathrm{CO}, \mathrm{USA})$ population.

Table 2. Genotype and allele distribution of FADS polymorphisms among 820 type 2 diabetes patients ${ }^{a}$.

\begin{tabular}{|c|c|c|c|c|c|c|}
\hline Gene & SNPs & $\begin{array}{c}\text { Alleles } \\
\text { (Major/Minor) }\end{array}$ & $\begin{array}{c}\text { Genotype } \\
(\mathrm{MM} / \mathrm{Mm} / \mathrm{mm})\end{array}$ & $\begin{array}{l}\text { Allele Frequency } \\
\qquad(\mathrm{M} / \mathrm{m})\end{array}$ & $\begin{array}{l}\text { Hapmap Genotype } \\
\text { Frequency } \\
\text { (Allele Frequency) }\end{array}$ & $\begin{array}{c}\text { HWE } \\
(p \text { Value })^{\mathrm{e}}\end{array}$ \\
\hline FADS1 & rs174547 & $\mathrm{T} / \mathrm{C}$ & $\begin{array}{c}280 / 395 / 145 \\
(34.1 / 48.2 / 17.7)\end{array}$ & $\begin{array}{c}955 / 685 \\
(58.2 / 41.8)\end{array}$ & $\begin{array}{c}33.3 / 41.7 / 25.0^{c} \\
(54.2 / 45.8)^{c}\end{array}$ & 0.774 \\
\hline FADS1 & rs174548 & $\mathrm{G} / \mathrm{C}$ & $\begin{array}{c}268 / 404 / 147 \\
(32.7 / 49.3 / 17.9)\end{array}$ & $\begin{array}{c}940 / 698 \\
(57.4 / 42.6)\end{array}$ & $\begin{array}{c}31.8 / 41.2 / 27.1^{\mathrm{c}} \\
(52.4 / 47.6)^{\mathrm{c}}\end{array}$ & 0.806 \\
\hline FADS2 & rs174575 & $\mathrm{C} / \mathrm{G}$ & $\begin{array}{c}613 / 188 / 19 \\
(74.8 / 22.9 / 2.3)\end{array}$ & $\begin{array}{c}1414 / 226 \\
(86.2 / 13.8)\end{array}$ & $\begin{array}{c}80.0 / 17.8 / 2.2^{\mathrm{d}} \\
(88.9 / 11.1)^{\mathrm{d}}\end{array}$ & 0.314 \\
\hline FADS2 & rs174576 & $\mathrm{A} / \mathrm{C}$ & $\begin{array}{c}280 / 397 / 143 \\
(34.1 / 48.1 / 17.4)\end{array}$ & $\begin{array}{c}957 / 683 \\
(58.3 / 41.7)\end{array}$ & $\begin{array}{c}31.8 / 43.5 / 24.7^{\mathrm{c}} \\
(53.5 / 46.5)^{\mathrm{c}}\end{array}$ & 0.911 \\
\hline FADS2 & rs 2727270 & $\mathrm{C} / \mathrm{T}$ & $\begin{array}{c}243 / 421 / 156 \\
(29.6 / 51.3 / 19.0)\end{array}$ & $\begin{array}{c}907 / 733 \\
(55.3 / 44.7)\end{array}$ & $\begin{array}{c}65.1 / 25.6 / 9.3^{\mathrm{d}} \\
(77.9 / 22.1)^{\mathrm{d}}\end{array}$ & 0.270 \\
\hline
\end{tabular}

Abbreviations: SNP, single nucleotide polymorphism; M, major allele; $\mathrm{m}$, minor allele. ${ }^{\text {a }}$ Data is presented as $\mathrm{n}(\%)$.

$\mathrm{b}$ The Hapmap website (http:/ / www.ncbi.nlm.nih.gov.) provides human genome information based on a general healthy population. ${ }^{c}$ The HapMap reference was provided by Chinese in Metropolitan Denver (CHD), CO, USA. d SNPs of rs174550, rs174575 and rs2727270 were not available from the Chinese Han population in Denver but from Han Chinese in Beijing (HCB), China. ${ }^{e}$ HWE, Hardy-Weinberg equilibrium. HWE for genotype frequency was analyzed by Chi-squared test.

Table 3. The differences of fatty acid desaturase activities among FADS1 and FADS2 genotypes in type 2 diabetes patients $(n=176)^{\text {a }}$.

\begin{tabular}{|c|c|c|c|c|c|c|}
\hline \multirow[t]{2}{*}{ Genotypes } & & $n$ & $\begin{array}{l}\text { D5D Activity } \\
(20: 4 \text { n6/20:3 n6) }\end{array}$ & $\begin{array}{l}\text { D6D Activity } \\
(18: 3 \mathrm{n} 6 / 18: 2 \mathrm{n} 6)\end{array}$ & $\begin{array}{l}\text { n-3 Pathway Activity } \\
\text { (20:5 n3/18:3 n3) }\end{array}$ & $\begin{array}{l}\text { n-6 Pathway Activity } \\
\text { (20:4 n6/18:2 n6) }\end{array}$ \\
\hline & & & \multicolumn{4}{|c|}{ Mean \pm SD } \\
\hline FADS1 & TT & 23 & $4.785 \pm 1.162$ & $0.020 \pm 0.010$ & $1.001 \pm 0.297$ & $0.389 \pm 0.087$ \\
\hline \multirow[t]{4}{*}{$\mathrm{rs} 174547^{\mathrm{d}}$} & $\mathrm{TC}$ & 90 & $4.188 \pm 1.306$ & $0.011 \pm 0.006$ & $0.947 \pm 0.358$ & $0.300 \pm 0.091$ \\
\hline & CC & 63 & $4.126 \pm 1.845$ & $0.006 \pm 0.005$ & $0.842 \pm 0.987$ & $0.233 \pm 0.075$ \\
\hline & $p$ value & & 0.05 & $<0.001$ & 0.001 & $<0.001$ \\
\hline & $p$ for trend & & 0.022 & $<0.001$ & $<0.001$ & $<0.001$ \\
\hline FADS2 & $\mathrm{CC}$ & 132 & $4.285 \pm 1.503$ & $0.011 \pm 0.008$ & $0.936 \pm 0.716$ & $0.292 \pm 0.983$ \\
\hline \multirow[t]{3}{*}{$\mathrm{rs} 174575^{\mathrm{d}}$} & $\mathrm{CG}+\mathrm{GG}$ & 44 & $4.120 \pm 1.552$ & $0.009 \pm 0.007$ & $0.858 \pm 0.407$ & $0.266 \pm 0.095$ \\
\hline & $p$ value ${ }^{\mathrm{b}}$ & & 0.426 & 0.008 & 0.309 & 0.098 \\
\hline & $p$ for trend ${ }^{c}$ & & - & - & - & - \\
\hline FADS2 & GG & 149 & $4.199 \pm 1.393$ & $0.011 \pm 0.008$ & $0.935 \pm 0.678$ & $0.291 \pm 0.094$ \\
\hline \multirow[t]{3}{*}{ rs498793 d } & $\mathrm{GA}+\mathrm{AA}$ & 27 & $4.490 \pm 2.069$ & $0.008 \pm 0.006$ & $0.815 \pm 0.479$ & $0.255 \pm 0.113$ \\
\hline & $p$ value ${ }^{\mathrm{b}}$ & & 0.866 & 0.031 & 0.112 & 0.065 \\
\hline & $p$ for trend ${ }^{c}$ & & - & - & - & - \\
\hline FADS2 & $\mathrm{CC}$ & 41 & $4.528 \pm 1.300$ & $0.015 \pm 0.009$ & $1.039 \pm 0.392$ & $0.355 \pm 0.101$ \\
\hline \multirow[t]{4}{*}{ rs $2727270^{d}$} & $\mathrm{CT}$ & 99 & $4.210 \pm 1.477$ & $0.011 \pm 0.007$ & $0.923 \pm 0.803$ & $0.275 \pm 0.090$ \\
\hline & $\mathrm{TT}$ & 36 & $4.013 \pm 1.802$ & $0.005 \pm 0.002$ & $0.759 \pm 0.321$ & $0.232 \pm 0.069$ \\
\hline & $p$ value & & 0.120 & $<0.001$ & 0.006 & $<0.001$ \\
\hline & $p$ for trend & & 0.039 & $<0.001$ & 0.001 & $<0.001$ \\
\hline
\end{tabular}

Abbreviations: $F A D S$, fatty acid desaturase; ${ }^{a}$ Data presented is mean \pm SD. ANOVA analysis was performed to test the differences among the FADS SNP genotypes ( $p$ value). Simple linear regression analysis was performed to test the trend among FADS SNP genotypes ( $p$ for trend). ${ }^{b}$ Independent $t$-test analysis was performed to test the differences for SNPs rs174575 and rs498793 genotypes ( $p$ value). ${ }^{c}$ Simple linear regression analysis ( $p$ for trend) didn't perform as the genotype combined into two groups. ${ }^{\mathrm{d}}$ rs174547, rs174575, rs498793 and rs2727270 are tag SNPs. 
Table 4. Differences of plasma lipid concentrations among FADS1 and FADS2 genotypes in type 2 diabetes patients $(n=176){ }^{\text {a }}$

\begin{tabular}{|c|c|c|c|c|c|c|c|}
\hline \multirow[b]{2}{*}{ Genotypes } & \multicolumn{7}{|c|}{ Plasma Lipids } \\
\hline & & & $\begin{array}{l}\text { Triglyceride } \\
\text { (mmol/L) }\end{array}$ & $\begin{array}{l}\text { Cholesterol } \\
\text { (mmol/L) }\end{array}$ & $\begin{array}{l}\text { LDL-C } \\
(\mathrm{mmol} / \mathrm{L})\end{array}$ & $\begin{array}{l}\text { HDL-C } \\
(\mathrm{mmol} / \mathrm{L})\end{array}$ & $\begin{array}{c}\text { Non-HDL-C } \\
(\mathrm{mmol} / \mathrm{L})\end{array}$ \\
\hline & & $n$ & & & Mean \pm SD & & \\
\hline \multirow{5}{*}{$\begin{array}{l}\text { rs1745 I } \\
\text { rs }\end{array}$} & TT & 23 & $1.4 \pm 0.6$ & $5.1 \pm 0.9$ & $3.3 \pm 0.9$ & $1.1 \pm 0.3$ & $4.0 \pm 0.9$ \\
\hline & $\mathrm{TC}$ & 90 & $1.6 \pm 1.2$ & $4.8 \pm 0.8$ & $3.0 \pm 0.7$ & $1.0 \pm 0.2$ & $3.8 \pm 0.8$ \\
\hline & CC & 63 & $1.7 \pm 1.1$ & $4.7 \pm 1.0$ & $2.9 \pm 0.9$ & $1.0 \pm 0.3$ & $3.7 \pm 0.9$ \\
\hline & $p$ value & & 0.699 & 0.201 & 0.098 & 0.098 & 0.485 \\
\hline & $p$ for trend & & 0.426 & 0.082 & 0.059 & 0.031 & 0.248 \\
\hline FADS2 & CC & 132 & $1.5 \pm 1.0$ & $4.8 \pm 0.9$ & $3.0 \pm 0.8$ & $1.0 \pm 0.3$ & $3.8 \pm 0.9$ \\
\hline \multirow[t]{3}{*}{$\operatorname{rs} 174575^{d}$} & $\mathrm{CG}+\mathrm{GG}$ & 44 & $1.7 \pm 1.3$ & $4.9 \pm 0.9$ & $3.0 \pm 0.9$ & $1.0 \pm 0.3$ & $3.8 \pm 0.9$ \\
\hline & $p$ value $^{\mathrm{b}}$ & & 0.625 & 0.645 & 0.983 & 0.964 & 0.627 \\
\hline & $p$ for trend ${ }^{c}$ & & - & - & - & - & - \\
\hline FADS2 & GG & 149 & $1.6 \pm 1.2$ & $4.9 \pm 0.9$ & $3.0 \pm 0.8$ & $1.0 \pm 0.3$ & $3.8 \pm 0.9$ \\
\hline \multirow{3}{*}{$\mathrm{rs} 498793^{\mathrm{d}}$} & $\mathrm{GA}+\mathrm{AA}$ & 27 & $1.4 \pm 0.7$ & $4.5 \pm 0.9$ & $2.8 \pm 0.8$ & $1.0 \pm 0.2$ & $3.5 \pm 0.8$ \\
\hline & $p$ value $^{\mathrm{b}}$ & & 0.482 & 0.034 & 0.261 & 0.298 & 0.096 \\
\hline & $p$ for trend ${ }^{c}$ & & - & - & - & - & - \\
\hline FADS2 & $\mathrm{CC}$ & 41 & $1.5 \pm 1.3$ & $5.0 \pm 0.9$ & $3.1 \pm 0.9$ & $1.1 \pm 0.3$ & $3.9 \pm 0.9$ \\
\hline \multirow[t]{4}{*}{ rs $2727270^{d}$} & $\mathrm{CT}$ & 99 & $1.6 \pm 0.9$ & $4.8 \pm 0.8$ & $3.0 \pm 0.7$ & $1.0 \pm 0.3$ & $3.8 \pm 0.8$ \\
\hline & TT & 36 & $1.6 \pm 1.3$ & $4.6 \pm 1.0$ & $3.0 \pm 1.0$ & $1.0 \pm 0.3$ & $3.7 \pm 1.0$ \\
\hline & $p$ value & & 0.472 & 0.147 & 0.249 & 0.083 & 0.354 \\
\hline & $p$ for trend & & 0.699 & 0.059 & 0.112 & 0.025 & 0.299 \\
\hline
\end{tabular}

Abbreviations: LDL-C, low-density lipoprotein cholesterol; HDL-C, high density lipoprotein cholesterol. ${ }^{\text {a }}$ Data presented is mean $\pm \mathrm{SD}$. ANOVA analysis was performed to test the differences among the FADS SNP genotypes ( $p$ value). Simple linear regression analysis was performed to test the trend among FADS SNP genotypes ( $p$ for trend).

$\mathrm{b}$ Independent $t$-test analysis was performed to test the differences for SNPs rs174575 and rs498793 genotypes ( $p$ value). ${ }^{c}$ Simple linear regression analysis ( $p$ for trend) didn't perform as the genotype combined into two groups.

d rs174547, rs174575, ,rs498793 and rs2727270 are tag SNPs.

As shown in Figure 1, tag SNP rs174547 was in high LD with rs174548 $\left(r^{2}=0.96\right)$, rs174550 $\left(r^{2}=0.99\right)$, rs174576 $\left(r^{2}=0.97\right)$ and $\operatorname{rs} 174583\left(r^{2}=0.97\right)$. Therefore, only four tag SNPs including rs174547, rs174575, rs2727270 and rs498793 were examined for their impact on desaturase activities (Table 3) and lipid status (Table 4).

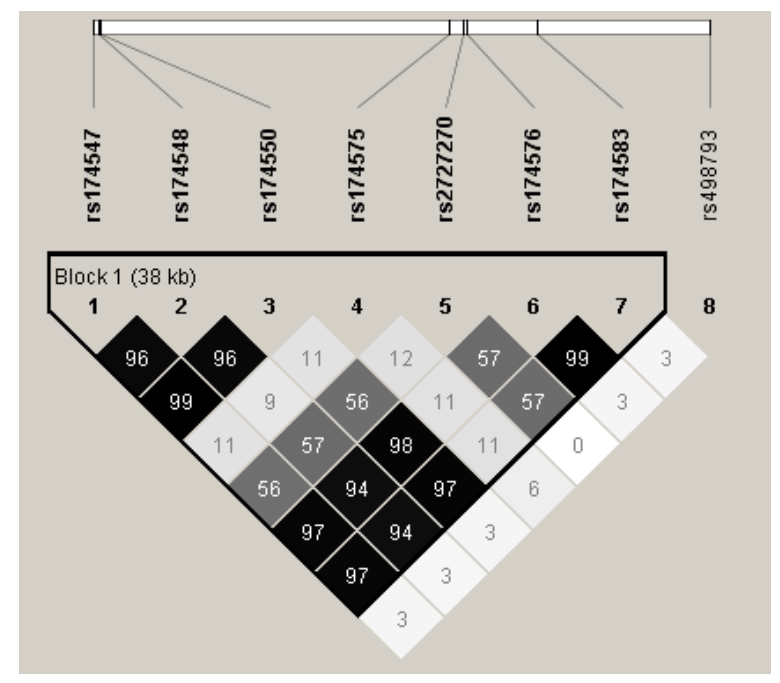

Figure 1. Linkage disequilibrium (LD) block generated by the 8 FADS1 and FADS2 polymorphisms genotyped, with pairwise $r^{2}$ values and color scheme. 


\subsection{FADS1 and FADS2 Genotypes and D5D and D6D Activities in Type 2 Diabetes Patients}

Table 3 presents the desaturase activities categorized by FADS genotypes of 176 type 2 diabetes patients not using lipid-lowering drugs. The FADS1 rs174547 TT, TC, CC genotypes were significantly and linearly associated with reduced D5D $(P$ for trend $=0.022)$ and D6D $(p$ for trend $<0.001)$ activities, as well as with n-3 and n-6 pathway activities (both $p$ for trend $<0.001$ ). Similarly, FADS2 rs2727270 CC, $\mathrm{CT}$ and TT genotypes were negatively correlated with D5D ( $p$ for trend $=0.039), \mathrm{D} 6 \mathrm{D}(p$ for trend $<0.001)$, $\mathrm{n}-3$ pathway ( $p$ for trend $=0.001$ ) and $\mathrm{n}-6$ pathway activities ( $p$ for trend $<0.001)$. Subjects carrying rs174575 and rs498793 minor allele were quite few, so genotypes with minor allele were combined for analysis. Subjects with rs174575 CG and GG genotypes $(p=0.008)$ as well as with rs498793 GA and AA genotypes $(p=0.031)$ had significantly lower D6D activities.

\subsection{FADS1 and FADS2 Genotypes and Lipid Traits in Type 2 Diabetes Patients}

Table 4 presents the plasma lipid profiles among FADS2 genotypes in 176 type 2 diabetes patients not using lipid-lowering drugs. The FADS1 rs174547 TT, TC, CC ( $p$ for trend $=0.031$ ) genotypes were negatively associated with reduced HDL-C concentrations. Similarly, FADS2 rs2727270 CC, CT and TT ( $p$ for trend $=0.025$ ) genotypes were negatively correlated with HDL-C levels. Frequencies of rs498793 A allele were small, so genotypes with minor allele were combined for analysis. Subjects carrying rs498793 GA and AA genotypes $(4.5 \pm 0.9 \mathrm{mmol} / \mathrm{L})$ were found to have significantly lower $(p=0.034)$ cholesterol levels than those with GG genotype $(4.9 \pm 0.9 \mathrm{mmol} / \mathrm{L})$. The FADS1 rs174575 did not appear to correlate with any lipid trait.

\subsection{Relations among FADS Genotypes, Desaturases Activities and HDL-C Levels}

Table 5 shows the independent associations among FADS1 rs174547, FADS2 rs2727270, desaturase activities and HDL-C concentrations in 176 diabetes patients not using statins or fibrates by multiple linear regression analysis. After adjustment for age, gender, body mass index and diabetes duration, $\mathrm{HbA1c}$, alcohol use and exercise habit, model 5 showed that D5D correlated significantly $(p=0.006)$ and D6D was marginally correlated $(p=0.07)$ with increased HDL-C. Whereas rs174547 and rs2727270 polymorphisms were not. We found D5D activities to correlate positively with HDL-C levels and D6D correlate marginally. FADS1 rs174547 and FADS2 rs2727270 polymorphisms were not found to correlate with those levels.

Table 5. Associations among FADS1 rs174547, FADS2 rs2727270, desaturases activities and HDL-C levels type 2 diabetes using multiple linear regression analysis $(n=176){ }^{\text {a }}$

\begin{tabular}{|c|c|c|c|c|c|c|c|c|c|c|}
\hline & \multicolumn{2}{|c|}{ Model 1} & \multicolumn{2}{|c|}{ Model 2} & \multicolumn{2}{|c|}{ Model 3} & \multicolumn{2}{|c|}{ Model 4} & \multicolumn{2}{|c|}{ Model 5} \\
\hline & Beta (SE) & $p$ & Beta (SE) & $p$ & Beta (SE) & $p$ & Beta (SE) & $p$ & Beta (SE) & $p$ \\
\hline rs174547 & $\begin{array}{c}-0.059 \\
(0.031)\end{array}$ & 0.056 & - & & - & & - & & $\begin{array}{c}0.006 \\
(0.050)\end{array}$ & 0.898 \\
\hline rs2727270 & - & & $\begin{array}{l}-0.057 \\
(0.031)\end{array}$ & 0.064 & - & & - & & $\begin{array}{l}-0.018 \\
(0.045)\end{array}$ & 0.687 \\
\hline D5D activity & - & & - & & $\begin{array}{c}0.034 \\
(0.014)\end{array}$ & 0.019 & - & & $\begin{array}{c}0.041 \\
(0.015)\end{array}$ & 0.006 \\
\hline D6D activity & - & & - & & - & & $\begin{array}{c}5.303 \\
(2.759)\end{array}$ & 0.056 & $\begin{array}{c}6.239 \\
(3.414)\end{array}$ & 0.070 \\
\hline
\end{tabular}

Abbreviations: D5D, delta-5-desaturase; D6D, delta-6-desaturase; ${ }^{a}$ All models are adjusted for confounding factors including age, gender, body mass index, education ( $\leq 6 \mathrm{y},>6 \mathrm{y}$ ), diabetes duration (years), hemoglobinA1c, alcohol use (yes, no) and exercise (yes, no).

\section{Discussion}

This study found FADS1 rs174547 and FADS2 rs2727270 genotypes were significantly correlated with decreased D5D and D6D activities and decreased HDL-C concentrations in patients with type 2 diabetes. Since rs174576, rs174548, rs174550 and rs174583 were in strong LD with the tag SNP rs174547, 
similar significant results were found. Two other studies conducted in Caucasians [13] and Asians [15] have reported SNP of rs174547 in FADS1 and rs174576 in FADS2 were in high LD $\left(r^{2} \geq 0.8\right)$.

Several earlier studies investigating healthy individuals have also shown that HDL-C concentration can be modified by rs $174547 \mathrm{C}$ allele in Japanese [14] and Koreans [26], and by rs174547 C allele and rs174550 T allele of FADS1 in people of Han Chinese decent [16]. The frequency of rs174547 C allele in our investigation was 0.418, 0.458 in the HapMap Chinese population (Table 2), and 0.31-0.51 in Japanese, Mongolians and Caucasians [11,14]. Furthermore, a genome-wide association study recruiting 19,840 European participants [11] found the minor $C$ allele to be associated with reduced HDL-C $\left(p=2.0 \times 10^{-12}\right)$ and increased triglyceride $\left(p=2.0 \times 10^{-14}\right)$. In that study, the authors measured RNA expression in over 39,000 transcripts from 957 human liver tissues. Performing expression quantitative trait locus analyses, they found the major $\mathrm{T}$ allele corresponded to the higher transcript levels $\left(p\right.$ value $\left.(F A D S 1)=5.0 \times 10^{-35}, p(F A D S 3)=1.0 \times 10^{-8}\right)$. Together, these findings show that SNP of rs174547 impacts both HDL-C levels and mRNA abundance of FADS1, suggesting that rs174547 SNP is either functional or in LD with a functional SNP affecting expression or activities of the FADS1.

Epidemiological studies have previously estimated D5D and D6D activities using PUFA product-to-precursor ratios. Our study found a reverse relationship between carriers of $C$ alleles of FADS1 rs174547 and ratios of 20:4 (n-6)/20:3 (n-6) and 18:3 (n-6)/18:2 (n-6) as D5D and D6D desaturase activities as well as 20:4 (n-6)/18:2 (n-6) and 20:5 (n-3)/18:3 (n-3) as n-6 and n-3 pathway activities. Our findings were also confirmed by recent findings of a negative association between rs $174547 \mathrm{C}$ allele and reduced desaturase activities or long chain PUFA levels in Dutch [27], in Europeans participating in the HELENA study [24], in Caucasians and Asians participating in Toronto Nutrigenomics and Health study [15] and in elderly Japanese [28]. Although none of these studies directly analyzed desaturase activities, their findings suggest that the $C$ allele of rs174547 may be related to insufficient endogenous conversion of PUFA from their precursors via D5D or D6D.

The biological mechanism underlying how FADS genetic variations interact with desaturase activities or PUFAs on HDL-C concentrations remains unclear. It is possible that $\mathrm{C}$ alleles of FADS1 rs174547 may impair desaturases leading to low yields of n-6 and n-3 long chain PUFAs such as 20:4n-6 and 20:5n-3. Furthermore, PUFAs are also known as ligands for peroxisome proliferator activating receptor $\alpha$, and they might modulate HDL-C metabolism by regulating expression of lipoprotein lipase and apolipoprotein A-I [29] A-II [30] and C-III [31].

This study found D5D activity was significantly correlated and D6D was marginally with HDL-C levels, but FADS1 rs174547and FADS2 rs2727270 polymorphisms were not, after adjusting for confounding factors. Measurements of plasma n-6 or n-3 PUFA concentrations status represent overall status of PUFA derived from both endogenous synthesis of PUFA via desaturation and elongation as well as exogenous dietary consumption. Although there is evidence that genes for these steps can be regulated by dietary PUFA, the degree to which dietary regulation of longer chain PUFA levels in tissues through changes in expression of desaturase and elongase genes is unclear. Thus, a study based on a larger diabetic sample size is needed to further explore possible interaction between dietary intake of PUFA and genetic variations in FADS affecting lipoprotein metabolism. Environmental exposure from diet and smoking is known to impinge on cardiovascular metabolic traits [32]. One study explored the relationship between FADS1/FADS2 SNPs and lipoprotein concentrations in two genetically similar Asian ethnic groups-Japanese $(n=21,004)$ and Mongolia $(n=1203)$ with distinctly different lifestyles [14]. That study found a relationship between C alleles of FADS1 rs174547 and reduced HDL-C and triglyceride levels in Japanese, but not in Mongolians. Substantial higher plasma n-3 PUFA and higher n-3/n-6 PUFA ratio was observed in Japanese [33] due to the fact that Japanese habitually consume large amounts of fish while Mongolians mainly consume livestock products, which may partly explain the inconsistent impact of rs174547 on blood lipids between these two groups [14]. Hellstrand et al. [10] reported an association between high 18:3n-3/18:2n- 6 ratio and $0.04 \mathrm{mmol} / \mathrm{L}$ and $0.02 \mathrm{mmol} / \mathrm{L}$ higher HDL-C in 4635 Swedish individuals with CC genotype $(p=0.046)$ and TC 
genotype ( $p=0.02)$, but not in those with TT genotype $(p=0.96)$. Their study results indicate that intake levels of n-3/n-6 PUFA modify the associations between genetic variation in FADS and HDL-C.

In this study, we did not use multiple test correction to adjust significance of $p$ value. While it would be ideal if we could use multiple testing correction to ensure robustness of our analysis, it may be limited in the case of this study because the hypotheses tested in this study were tested by analyses of the a priori, not post hoc, and because we had many comparisons to perform, it would render ideal $p$ value indication of significance too small to be achieved. Our study is limited in that our sample was small and our results may not be generalized to diabetes populations of other ethnicities. Future studies need to increase sample sizes of diabetes patient groups and include a healthy controls in order to better examine the relative impacts of FADS genetic polymorphisms as well as PUFA status on dyslipidemia in diabetes and healthy subjects.

\section{Conclusions}

In conclusion, FADS1 rs174547 and FADS2 rs2727270 genotypes were significantly correlated with decreased HDL-C concentrations, and D5D /D6D activities as estimated as 20:4(n-6)/20:3 (n-6) and 18:3 (n-6)/18:2 (n-6) in a linear pattern in patients with type 2 diabetes. The results of our multiple variable regression analysis also further revealed that higher HDL-C status levels were positively correlated with higher D5D and marginally with D6D activities, but not with the polymorphisms of FADS1 rs174547and FADS2 rs2727270. Future studies with greater sample sizes are needed to confirm this finding and further explore whether desaturase activities or PUFA status could interact with FADS genetic variants to affect lipid metabolism or cardiovascular disease development in type 2 diabetes patients. The understanding of the interaction between environmental modifiers and genetic variants may facilitate future development of preventative strategies for public health genomics and counseling or treatment approaches for personalized medicine.

Acknowledgments: We are grateful to all research nurses and health professionals for their assistance and grateful to all the subjects who participated in this investigation. This study was supported by grants from the Taiwan National Health Research Institute (NCT00288678), Taiwan National Science Council (NSC 102-2628B-037-008-MY3) and Kaohsiung Medical University (KMUH104-4R71).

Author Contributions: Meng-Chuan Huang and Wen-Tsan Chang wrote the manuscript. Hsin-Yu Chang, Hsin-Fang Chung and Ya-Fang Huang assisted in recruitment of subjects and performed the experiments. Meng-Chuan Huang, Hsin-Yu Chang, Hsin-Fang Chung and Fang-Pei Chen performed statistical analysis and interpreted the results. Meng-Chuan Huang, Shang-Jyh Hwang and Chih-Cheng Hsu conceived and designed the study. Meng-Chuan Huang, Wen-Tsan Chang, and Chih-Cheng Hsu reviewed and revised the manuscript. All authors read and approved the final manuscript.

Conflicts of Interest: The authors declare that there is no conflict of interests.

\section{Abbreviations}

The following abbreviations are used in the manuscript:

$\begin{array}{ll}\text { ANOVA } & \text { Analysis and analysis of variance } \\ \text { D5D } & \text { Delta-5 desaturase } \\ \text { D6D } & \text { Delta-6 desaturase } \\ \text { PUFA } & \text { Polyunsaturated fatty acids } \\ \text { FADS } & \text { Fatty acid desaturase (FADS) } \\ \text { HDL-C } & \text { High density lipoprotein-cholesterol } \\ \text { HWE } & \text { Weinberg equilibrium expectation } \\ \text { LDL-C } & \text { Low density lipoprotein-cholesterol } \\ \text { LD } & \text { Linkage disequilibrium } \\ \text { MAF } & \text { Minor allele frequencies } \\ \text { SNP } & \text { single nucleotide polymorphisms }\end{array}$




\section{References}

1. Quispe, R.; Martin, S.S.; Jones, S.R. Triglycerides to high-density lipoprotein-cholesterol ratio, glycemic control and cardiovascular risk in obese patients with type 2 diabetes. Curr. Opin. Endocrinol. Diabetes Obes. 2016, 23, 150-156. [CrossRef] [PubMed]

2. Siebel, A.L.; Heywood, S.E.; Kingwell, B.A. HDL and glucose metabolism: Current evidence and therapeutic potential. Front. Pharmacol. 2015, 6, 258. [CrossRef] [PubMed]

3. Verges, B. Pathophysiology of diabetic dyslipidaemia: Where are we? Diabetologia 2015, 58, 886-899. [CrossRef] [PubMed]

4. In the clinic. Type 2 diabetes. Ann. Intern. Med. 2007, 146, ITC1.

5. Marquardt, A.; Stohr, H.; White, K.; Weber, B.H. CDNA cloning, genomic structure, and chromosomal localization of three members of the human fatty acid desaturase family. Genomics 2000, 66, 175-183. [CrossRef] [PubMed]

6. Schaeffer, L.; Gohlke, H.; Muller, M.; Heid, I.M.; Palmer, L.J.; Kompauer, I.; Demmelmair, H.; Illig, T.; Koletzko, B.; Heinrich, J. Common genetic variants of the FADS1 FADS2 gene cluster and their reconstructed haplotypes are associated with the fatty acid composition in phospholipids. Hum. Mol. Genet. 2006, 15, 1745-1756. [CrossRef] [PubMed]

7. Lattka, E.; Illig, T.; Koletzko, B.; Heinrich, J. Genetic variants of the FADS 1 FADS 2 gene cluster as related to essential fatty acid metabolism. Curr. Opin. Lipidol. 2010, 21, 64-69. [CrossRef] [PubMed]

8. Cormier, H.; Rudkowska, I.; Thifault, E.; Lemieux, S.; Couture, P. Polymorphisms in fatty acid desaturase (FADS) gene cluster: Effects on glycemic controls following an omega-3 polyunsaturated fatty acids (PUFA) supplementation. Genes 2013, 10, 485-498. [CrossRef] [PubMed]

9. Tanaka, T.; Shen, J.; Abecasis, G.R.; Kisialiou, A.; Ordovas, J.M.; Guralnik, J.M.; Singleton, A.; Bandinelli, S.; Cherubini, A.; Arnett, D.; et al. Genome-wide association study of plasma polyunsaturated fatty acids in the Inchianti study. PLoS Gene 2009, 5, e1000338. [CrossRef] [PubMed]

10. Hellstrand, S.; Sonestedt, E.; Ericson, U.; Gullberg, B.; Wirfalt, E.; Hedblad, B.; Orho-Melander, M. Intake levels of dietary long-chain pufas modify the association between genetic variation in FADS and LDL-C. J. Lipid Res. 2012, 53, 1183-1189. [CrossRef] [PubMed]

11. Kathiresan, S.; Willer, C.J.; Peloso, G.M.; Demissie, S.; Musunuru, K.; Schadt, E.E.; Kaplan, L.; Bennett, D.; Li, Y.; Tanaka, T.; et al. Common variants at 30 loci contribute to polygenic dyslipidemia. Nat. Genet. 2009, 41, 56-65. [CrossRef] [PubMed]

12. Aulchenko, Y.S.; Ripatti, S.; Lindqvist, I.; Boomsma, D.; Heid, I.M.; Pramstaller, P.P.; Penninx, B.W.; Janssens, A.C.; Wilson, J.F.; Spector, T.; et al. Loci influencing lipid levels and coronary heart disease risk in 16 European population cohorts. Nat. Genet. 2009, 41, 47-55. [CrossRef] [PubMed]

13. Abdelmagid, S.A.; Clarke, S.E.; Roke, K.; Nielsen, D.E.; Badawi, A.; El-Sohemy, A.; Mutch, D.M.; Ma, D.W. Ethnicity, sex, FADS genetic variation, and hormonal contraceptive use influence delta-5- and delta-6-desaturase indices and plasma docosahexaenoic acid concentration in young Canadian adults: A cross-sectional study. Nutr. Metab. 2015, 12, 14. [CrossRef] [PubMed]

14. Nakayama, K.; Bayasgalan, T.; Tazoe, F.; Yanagisawa, Y.; Gotoh, T.; Yamanaka, K.; Ogawa, A.; Munkhtulga, L.; Chimedregze, U.; Kagawa, Y.; et al. A single nucleotide polymorphism in the FADS 1/ FADS 2 gene is associated with plasma lipid profiles in two genetically similar Asian ethnic groups with distinctive differences in lifestyle. Hum. Genet. 2010, 127, 685-690. [CrossRef] [PubMed]

15. Merino, D.M.; Johnston, H.; Clarke, S.; Roke, K.; Nielsen, D.; Badawi, A.; El-Sohemy, A.; Ma, D.W.; Mutch, D.M. Polymorphisms in FADS 1 and FADS 2 alter desaturase activity in young Caucasian and Asian adults. Mol. Genet. Metab. 2011, 103, 171-178. [CrossRef] [PubMed]

16. Zhu, J.; Sun, Q.; Zong, G.; Si, Y.; Liu, C.; Qi, Q.; Ye, X.; Sun, L.; Sheng, H.; Li, H.; et al. Interaction between a common variant in FADS 1 and erythrocyte polyunsaturated fatty acids on lipid profile in Chinese Hans. J. Lipid Res. 2013, 54, 1477-1483. [CrossRef] [PubMed]

17. Lu, Y.; Feskens, E.J.; Dolle, M.E.; Imholz, S.; Verschuren, W.M.; Muller, M.; Boer, J.M. Dietary n-3 and n-6 polyunsaturated fatty acid intake interacts with FADS 1 genetic variation to affect total and HDL-cholesterol concentrations in the doetinchem cohort study. Am. J. Clin. Nutr. 2010, 92, 258-265. [CrossRef] [PubMed] 
18. Vessby, B.; Tengblad, S.; Lithell, H. Insulin sensitivity is related to the fatty acid composition of serum lipids and skeletal muscle phospholipids in 70-year-old men. Diabetologia 1994, 37, 1044-1050. [CrossRef] [PubMed]

19. Martinelli, N.; Girelli, D.; Malerba, G.; Guarini, P.; Illig, T.; Trabetti, E.; Sandri, M.; Friso, S.; Pizzolo, F.; Schaeffer, L.; et al. FADS s genotypes and desaturase activity estimated by the ratio of arachidonic acid to linoleic acid are associated with inflammation and coronary artery disease. Am. J. Clin. Nutr. 2008, 88, 941-949. [PubMed]

20. American, D.A. Standards of medical care in diabetes. Diabetes Care 2004, 27 (Suppl. 1), S15-S35.

21. Hsu, C.C.; Chang, H.Y.; Huang, M.C.; Hwang, S.J.; Yang, Y.C.; Tai, T.Y.; Yang, H.J.; Chang, C.T.; Chang, C.J.; Li, Y.S.; et al. Association between insulin resistance and development of microalbuminuria in type 2 diabetes: A prospective cohort study. Diabetes Care 2011, 34, 982-987. [CrossRef] [PubMed]

22. Bligh, E.G.; Dyer, W.J. A rapid method of total lipid extraction and purification. Can. J. Biochem. Physiol. 1959, 37, 911-917. [CrossRef] [PubMed]

23. Chung, H.F.; Long, K.Z.; Hsu, C.C.; Al Mamun, A.; Jhang, H.R.; Shin, S.J.; Hwang, S.J.; Huang, M.C. Association of $\mathrm{n}-3$ polyunsaturated fatty acids and inflammatory indicators with renal function decline in type 2 diabetes. Clin. Nutr. 2015, 34, 229-234. [CrossRef] [PubMed]

24. Bokor, S.; Dumont, J.; Spinneker, A.; Gonzalez-Gross, M.; Nova, E.; Widhalm, K.; Moschonis, G.; Stehle, P.; Amouyel, P.; De Henauw, S.; et al. Single nucleotide polymorphisms in the FADS gene cluster are associated with delta- 5 and delta- 6 desaturase activities estimated by serum fatty acid ratios. J. Lipid Res. 2010, 51, 2325-2333. [CrossRef] [PubMed]

25. Tsai, M.H.; Lin, K.M.; Hsiao, M.C.; Shen, W.W.; Lu, M.L.; Tang, H.S.; Fang, C.K.; Wu, C.S.; Lu, S.C.; Liu, S.C.; et al. Genetic polymorphisms of cytochrome p450 enzymes influence metabolism of the antidepressant escitalopram and treatment response. Pharmacogenomics 2010, 11, 537-546. [CrossRef] [PubMed]

26. Park, M.H.; Kim, N.; Lee, J.Y.; Park, H.Y. Genetic loci associated with lipid concentrations and cardiovascular risk factors in the korean population. J. Med. Genet. 2011, 48, 10-15. [CrossRef] [PubMed]

27. Lu, Y.; Vaarhorst, A.; Merry, A.H.; Dolle, M.E.; Hovenier, R.; Imholz, S.; Schouten, L.J.; Heijmans, B.T.; Muller, M.; Slagboom, P.E.; et al. Markers of endogenous desaturase activity and risk of coronary heart disease in the carema cohort study. PLoS ONE 2012, 7, e41681. [CrossRef] [PubMed]

28. Horiguchi, S.; Nakayama, K.; Iwamoto, S.; Ishijima, A.; Minezaki, T.; Baba, M.; Kontai, Y.; Horikawa, C.; Kawashima, H.; Shibata, H.; et al. Associations between a fatty acid desaturase gene polymorphism and blood arachidonic acid compositions in Japanese elderly. Prostaglandins Leukot. Essent. Fatty Acids 2016, 105, 9-14. [CrossRef] [PubMed]

29. Vu-Dac, N.; Schoonjans, K.; Laine, B.; Fruchart, J.C.; Auwerx, J.; Staels, B. Negative regulation of the human apolipoprotein A-I promoter by fibrates can be attenuated by the interaction of the peroxisome proliferator-activated receptor with its response element. J. Biol. Chem. 1994, 269, 31012-31018. [PubMed]

30. Vu-Dac, N.; Schoonjans, K.; Kosykh, V.; Dallongeville, J.; Fruchart, J.C.; Staels, B.; Auwerx, J. Fibrates increase human apolipoprotein A-II expression through activation of the peroxisome proliferator-activated receptor. J. Clin. Investig. 1995, 96, 741-750. [CrossRef] [PubMed]

31. Hertz, R.; Bishara-Shieban, J.; Bar-Tana, J. Mode of action of peroxisome proliferators as hypolipidemic drugs. Suppression of apolipoprotein C-III. J. Biol. Chem. 1995, 270, 13470-13475. [CrossRef] [PubMed]

32. National Cholesterol Education Program Expert Panel on Detection, Evaluation; Treatment of High Blood Cholesterol in Adults. Third report of the National Cholesterol Education Program (NCEP) expert panel on detection, evaluation, and treatment of high blood cholesterol in adults (Adult Treatment Panel III) final report. Circulation 2002, 106, 3143-3421.

33. Nogi, A.; Yang, J.; Li, L.; Yamasaki, M.; Watanabe, M.; Hashimoto, M.; Shiwaku, K. Plasma n-3 polyunsaturated fatty acid and cardiovascular disease risk factors in Japanese, korean and mongolian workers. J. Occup. Health 2007, 49, 205-216. [CrossRef] [PubMed]

(C) 2017 by the authors. Licensee MDPI, Basel, Switzerland. This article is an open access article distributed under the terms and conditions of the Creative Commons Attribution (CC BY) license (http:/ / creativecommons.org/licenses/by/4.0/). 